\title{
Delay in Aging Process
}

\author{
Islam MA
}

\section{Ageing}

Ageing is the process of becoming older. In humans, ageing represents the accumulation of changes in a human being over time, ${ }^{1}$ encompassing physical, psychological, and social change. Reaction time, for example, may slow with age, while knowledge of world events and wisdom may expand. Ageing is among the greatest known risk factors for most human diseases: of the roughly 150,000 people who die each day across the globe, about two thirds die from age-related causes. In industrialized nations, the proportion is higher; reaching $90 \%{ }^{2}$ The causes of ageing are uncertain. Current theories are assigned to the damage concept, whereby the accumulation of damage such as DNA oxidation may cause biological systems to fail; or to the programmed ageing concept, whereby internal processes such as DNA methylation may cause ageing. The discovery, in 1934, that calorie restriction can extend lifespan by $50 \%$ in rats has motivated research into delaying and preventing ageing.

\section{Effects of ageing}

Effects of ageing are many, started as age progresses, varies in different ages and individuals. Teenagers lose the young child's ability to hear high-frequency sounds above $20 \mathrm{kHz}$. Some cognitive decline begins in the mid-20s Wrinkles develop mainly due to photoageing, particularly affecting sun-exposed areas (face). ${ }^{3}$ After peaking in the mid-20s, female fertility declines. People over 35 years old are at risk for developing presbyopia, and most people benefit from reading glasses by age 45-50. Age can result in visual impairment, whereby non-verbal communication is reduced, which can lead to isolation and possible depression. Macular degeneration causes vision loss and increases with age, affecting nearly $12 \%$ of those above the age of 80 . This degeneration is caused by systemic changes in the circulation of waste products and by growth of abnormal vessels around the retina. By age 80 , more than half of all Americans either have a cataract or have had cataract surgery. ${ }^{4}$ Hair turns grey with age. Pattern hair loss by the age of 50 affects about half of males and a quarter of females. ${ }^{5}$ Menopause typically occurs between 49 and 52 years of age. Around a third of people between 65 and 74 have hearing loss and almost half of people older than 75. ${ }^{6}$ In the 60-64 age cohort, the incidence of osteoarthritis rises to $53 \%$. Only $20 \%$ however report disabling osteoarthritis at this age. Frailty, defined as loss of muscle mass and mobility, affects $25 \%$ of those over $85 .^{7}$ Atherosclerosis is classified as an ageing disease. It leads to cardiovascular disease for example stroke and heart attack which globally is the most common cause of death. ${ }^{8,9}$ The maximum human lifespan is suggested to be 115 years "for the foreseeable future". ${ }^{10.11}$ Dementia becomes more common with age. About 3\% of people between the ages of 65-74 have dementia. 19\% between 75 and 84 and nearly half of those over 85 years of age. The spectrum includes mild cognitive impairment and the neurodegenerative diseases of Alzheimer's disease, cerebrovascular disease, Parkinson's disease and Lou Gehrig's disease.

Furthermore, many types of memory may decline with ageing, but not semantic memory or general knowledge such as vocabulary definitions, which typically increases or remains steady until late adulthood. Intelligence may decline with age, though the rate may vary depending on the type and may in fact remain steady throughout most of the lifespan, dropping suddenly only as people near the end of their lives. Individual variations in rate of cognitive decline may therefore be explained in terms of people having different lengths of life. There might be changes to the brain: after 20 years of age there may be a $10 \%$ reduction each decade in the total length of the brain's myelinated axons. $^{12,13}$

\section{1. *Dr. Md. Aminul Islam \\ Associate Professor \\ Department of Medicine \\ Community Based Medical College \\ Bangladesh, Mymensingh \\ Address of correspondence \\ Email: aminul1712@gmail.com \\ Mobile: +8801712116214}




\section{Prevention and delay}

Calorie restriction, amount of sleep, physical exercise, and avoidance of chronic stress, all of these are associated with longevity of life and delay or prevent age related diseases. There are some scientific evidences have been proven that few drugs and interventions have been shown to retard or reverse the biological effects of ageing

\section{Life style}

Caloric restriction substantially affects lifespan in many animals, including the ability to delay or prevent many age-related diseases. In rodents, this has been shown to increase lifespan by up to $50 \%$; similar effects occur for yeast and Drosophila. No lifespan data exist for humans on a calorie-restricted diet, but several reports support protection from age-related diseases. ${ }^{14}$ Researchers found that moderate calorie restriction rather than extreme calorie restriction is sufficient to produce the observed health and longevity benefits in the rhesus monkeys. The benefits of dietary restriction can also be found by changing the macro nutrient profile to reduce protein intake without any changes to calorie level, resulting in similar increases in longevity. ${ }^{15}$ The Mediterranean diet is credited with lowering the risk of heart disease and early death. The major contributors to mortality risk reduction appear to be a higher consumption of vegetables, fish, fruits, nuts and monounsaturated fatty acids, i.e., olive oil. ${ }^{16}$

The amount of sleep has an impact on mortality. People who live the longest report sleeping for six to seven hours each night. Lack of sleep ( $<5$ hours) more than doubles the risk of death from cardiovascular disease, but too much sleep ( $>9$ hours) is associated with a doubling of the risk of death, though not primarily from cardiovascular disease. Sleeping more than 7 to 8 hours per day has been consistently associated with increased mortality. ${ }^{17}$

Physical exercise may increase life expectancy. People who participate in moderate to high levels of physical exercise have a lower mortality rate compared to individuals who are not physically active. Moderate levels of exercise have been correlated with preventing aging and improving quality of life by reducing inflammatory potential. The majority of the benefits from exercise are achieved with around 3500 metabolic equivalent (MET) minutes per week. For example, climbing stairs 10 minutes, vacuuming 15 minutes, gardening 20 minutes, running 20 minutes, and walking or bicycling for 25 minutes on a daily basis would together achieve about 3000 MET minutes a week. $^{18,19,20}$

Avoidance of chronic stress is associated with a slower loss of telomeres in most but not all studies, and with decreased cortisol levels. A chronically high cortisol level compromises the immune system, causes cardiac damage/arterosclerosis and is associated with facial ageing, and the latter in turn is a marker for increased morbidity and mortality. Stress can be countered by social connection, spirituality, and for men more clearly than for women married life, all of which are associated with longevity. ${ }^{21}$

\section{Medical intervention}

Recent research and advances indicated that few drugs and interventions have been shown to retard or reverse the biological effects of ageing in animal models, but none has yet been proven to do so in humans. Among the experimental drugs metformin, resveratrol - a calorie mimetic and rapamycin have shown significant effect on ageing, mostly on animal models and prokaryotes and improvement in other cognitive and physical functions. ${ }^{22,23,24}$

Cancer geneticist Ronald A. DePinho and his colleagues published research in mice by genetically removed telomerase activity which causing premature ageing. Then they restored telomerase activity and found the mice were rejuvenated. ${ }^{25}$

As there are many simple techniques, for example, physical activity and caloric restriction can delay ageing and its effects, these are very much adaptable for the developing countries like us. We need preparedness programs for the elderly and practical approach for their management with low cost set up and more awareness programs to minimize age related diseases, disability and dependency to family members.

\section{References}

1. Bowen, Richard L.; Atwood, Craig S. (2004). Living and Dying for Sex. Gerontology. 50 (5): 265-90.

2. Dillin A, Gottschling DE, Nyström $T$ (2014). The good and the bad of being connected: the integrons of Aging. CurrOpin Cell Biol. 26: 107-12.

3. Thurstan SA, Gibbs NK, Langton AK, Griffiths CE, Watson $R E$, Sherratt MJ (2012). Chemical consequences of cutaneous photoageing. Chem Cent J. 6(1): 34.

4. Weale RA (2003). Epidemiology of refractive errors and presbyopia. SurvOphthalmol. 48 (5): 515-43. 
5. Pandhi, D; Khanna, D (2013). Premature graying of hair. Indian Journal of dermatology, venereology and leprology. 79 (5): 641-53.

6. Hearing Loss and Older Adults. National Institute on Deafness and Other Communication Disorders. Retrieved September 11, 2016.

7. Fried, LP; Tangen, $C M$; Walston, J; Newman, $A B$; Hirsch, $C$; Gottdiener, J; Seeman, T; Tracy, R; Kop, WJ; Burke, G; McBurnie, MA (Mar 2001). Frailty in older adults: evidence for a phenotype. The Journals of Gerontology. Series A, Biological Sciences and Medical Sciences. 56 (3): M146-56.

8. Wang JC, Bennett $M$ (2012). Aging and atherosclerosis: mechanisms, functional consequences, and potential therapeutics for cellular senescence. Circ Res. 111 (2): 245-59.

9. Herrington $W$, Lacey $B$, Sherliker $P$, Armitage J, Lewington $S$ (2016). Epidemiology of Atherosclerosis and the Potential to Reduce the Global Burden of Atherothrombolic Disease. Circ Res. 118 (4): 535-46.

10. Zimmer, Carl (October 5, 2016). What's the Longest Humans Can Live? 115 Years, New Study Says. New York Times. Retrieved October 6, 2016.

11. Dong, Xiao; Milholland, Brandon; Vijg, Jan (October 5, 2016) Evidence for a limit to human lifespan. Nature. 538 (7624): 257-259.

12. Marner, Lisbeth; Nyengaard, Jens R.; Tang, Yong; Pakkenberg, Bento (2003). Marked loss of myelinated nerve fibers in the human brain with ago. The Journal of Comparative Neurology. 462 (2): 144-52.

13. Peters, Alan (1 January 2007). Riddle, David R., ed. Brain Aging: Models, Methods, and Mechanisms CRC Press/Taylor \& Francis.

14. Guarente L, Picard F (2005). Calorie restriction-the SIR2 connection. Cell. 120 (4): 473-82.

15. Nakagawa, Shinichi; Lagisz, Malgorzata; Hector, Katie L.; Spencer, Hamish G. (2012-06-01). Comparative and metaanalytic insights into life extension via dietary restriction. Aging Cell. 11 (3): 401-409.

16. Sofi F, Cesari F, Abbate R, Gensini GF, Casini A (2008). Adherence to Mediterranean diet and health status: meta-analysis. BMJ (Clinical research ed.). 337 (sep 11 2): a1344.

17. Ferrie JE, Shipley MJ, Cappuccio FP, Brunner E, Miller MA, Kumari M. Marmot MG (December 2007). A prospective study of change in sleep duration: associations with mortality in the Whitehall II cohort. Sleep. 30 (12): 165966.

18. Gremeaux, V; Gayda, $M$; Lepers, $R$; Sosner, P; Junoau, $M$; Nigam, A (December 2012). Exercise and longevity Maturitas. 73 (4): 312-7.

19. Woods, Jeffrey A.; Wilund, Kenneth R.; Martin, Stephen A.; Kistler, Brandon M. (2011-10-29). Exercise, Inflammation and Aging. Aging and Disease. 3 (1): 130140.

20. Kyu, Hmwe H; Bachman, Victoria F; Alexander, Lily T; Mumford, John Everett; Alshin, Ashkan; Estep, Kara; Veerman, J Lennert; Delwiche, Kristen; lannarone, Marissa L; Moyer, Madeline L; Cercy, Kelly; Vos, Theo; Murray, Christopher JL; Forouzanfar, Mohammad H 19 August 2016). Physical activity and risk of breast cancer, colon cancer, diabetes, Ischemic heart disease, and ischemic stroke events: systematic review and dose-response metaanalysis for the Global Burden of Disease Study 2013. BMJ:i3867.

21. Quinlan J, Tu MT, Langlois EV, Kapoor M, Ziegler D, Fahmi $H$, Zunzunegui MV (2014). Protocol for a systematic review of the association between chronic stress during the life course and telomere length. Syst Rev (Review). 3 (40): 40.

22. Agarwal B, Baur JA (2011). Resveratrol and life extension. Ann NY Acad Sci. 1215 (1): 138-43.

23. Lam YY, Peterson CM, Ravussin E (2013). Resveratrol vs. calorie restriction: data from rodents to humans. Exp Gerontol.48 (10): 1018-24.

24. Pryor, R; Cabreiro, F (1 November 2015). Repurposing metformin: an old drug with now tricks in its binding pockets. The Biochemical Journal. 471 (3): 307-22. doi:10. 1042/bj20150497.

25. Callaway, Ewen. Telomerase reverses ageing process. Nature, 2010. 